\title{
DSP 4 You
}

Richard F. Lyon, Foveon Chief Scientist

W hether you think it means digital signal processing, or digital signal processor, DSP is a topic that affects your life, if not your work as a software engineer. Since 1979 saw the design of the first wave of user-programmable DSP chips-the Intel 2920, the NEC $\mu$ PD7720, and the Bell Labs (AT\&T) DSP-1-2004 is a 25th anniversary that's appropriately celebrated with the collection of articles presented in this month's ACM Queue.

The conversion of traditionally analog media to digital began slowly, with the invention of PCM (pulse code modulation) by Alec H. Reeves in 1937. The 1948 paper, "The Philosophy of PCM," by Barney Oliver, John Pierce, and Claude Shannon of Bell Labs, following on their use of PCM for secret wartime communications between Churchill and Roosevelt, laid out an amazing and farthinking view of why and how digital was going to take over the analog world. The rest, as they say, is history...

In my own signal processing career, I've had the pleasure of moving back and forth between analog and digital processing, and between hardware and software, several times. When I was doing digital, I cited the Bible's admonition to "Let your communication be, Yea, yea; Nay, nay: for whatsoever is more than these cometh of evil." And when I worked on analog, I lived by Launegayer's maxim, "All the world's an analog stage, and digital circuits play only bit parts." Inexorably, the success of DSP continues to create an increasing demand for both digital and ana$\log$ circuits for devices that interface with the real world.

Most of the articles in this issue start by reminding you that more and more, computing is about delivering and processing media signals digitally, so I won't belabor that point here. Let's cut to the issue: DSP algorithms, architectures, and programming are different in important ways from the other things that you may have been trained on as a software engineer. The authors of articles in this issue help you understand those differences, their implications, and some ways of dealing with them.

Pat Hays reviews the history of the digital signal processor in "DSPs: Back to the Future," emphasizing how application and technology constraints conspired to create a distinct type of processor, and how those constraints and architectures are evolving. As architect of several

\section{Computing is about DELIVERING AND PROCESSING MEDIA SIGNALS DIGITALLY. important DSPs, Hays is well placed to provide this survey. \\ Ray Simar and Gene Frantz provide a good} overview of DSP hardware approaches and algorithm characteristics, explaining not just the programmable DSP but also more dedicated ASIC (application-specific integrated circuit), ASSP (application-specific standard part), and FPGA (field-programmable gate array) approaches. Their article explores the relation of architecture properties, such as power and cost, to different types of DSP algorithms.

Homayoun Shahri discusses mapping DSP algorithms to DSP architectures. He discusses methodologies for choosing the "right" algorithm, hardware/software partitioning, verification, and mapping of algorithms in fixed as well as configurable DSPs, emphasizing optimization via source-code transformations.

The article on stream processors by William J. Dally and his coworkers presents a novel architecture that approaches the efficiency of ASIC signal processors while retaining programmability on DSP tasks. This is the most exciting new DSP architecture I've seen in a long time.

Boris Murmann and Bernhard Boser's article on digitally assisted analog ICs explains how intensive DSP can be applied to even primitive parts of a system, such as the internals of an analog-to-digital converter, to save power and cost relative to a more conventional design.

Finally, Stan Kelly-Bootle's Curmudgeon column, "Damnéd Digits," seems particularly appropriate to this issue. $Q$

RICHARD F. LYON is chief scientist and vice president of research at Foveon. His work involves processing the signals from Foveon $\mathrm{X} 3$ color image sensors into eye-popping digital photographs. He is known to the communities of VLSI (very large-scale integration) signal processing, speech and handwriting recognition, auditory models, and optical mice through his decades of work in the research labs of AT\&T, Xerox, Schlumberger, and Apple. Lyon is a fellow of IEEE and a spouse member of ACM, and has more than 35 issued U.S. patents. 